\title{
COLON CANCER
}

\section{A prospective comparative study of narrow-band imaging, chromoendoscopy, and conventional colonoscopy in the diagnosis of colorectal neoplasia}

\author{
Han-Mo Chiu, Chi-Yang Chang, Chien-Chuan Chen, Yi-Chia Lee, Ming-Shiang Wu, Jaw-Town Lin, \\ Chia-Tung Shun, Hsiu-Po Wang
}

See end of article for authors' affiliations

........ a.

Correspondence to: Dr H-P Wang, Department of Emergency Medicine, National Taiwan University Hospital, 7, Chung-Shan South Road, Taipei, Taiwan; whp@ha.mc.ntu.edu.tw

Received 20 April 2006 Accepted 5 September 2006 Published Online First 27 September 2006

\begin{abstract}
Background: Discrimination between neoplastic and non-neoplastic lesions is crucial in colorectal cancer screening. Application of narrow-band imaging (NBI) in colonoscopy visualises mucosal vascular networks in neoplastic lesions and may improve diagnostic accuracy.

Aim: To compare the diagnostic efficacy of NBI in differentiating neoplastic from non-neoplastic colorectal lesions with diagnostic efficacies of standard modalities, conventional colonoscopy, and chromoendoscopy. Methods: In this prospective study, 180 colorectal lesions from 133 patients were observed with conventional colonoscopy, and under low-magnification and high-magnification NBI and chromoendoscopy. Lesions were resected for histopathological analysis. Endoscopic images were stored electronically and randomly allocated to two readers for evaluation. Sensitivity, specificity and diagnostic accuracy of each endoscopic modality were assessed by reference to histopathology.

Results: NBI and chromoendoscopy scored better under high magnification than under low magnification in comparison with conventional colonoscopy. The diagnostic accuracy of NBI with low or high magnification was significantly higher than that of conventional colonoscopy (low magnification: $p=0.0434$ for reader 1 and $p=0.004$ for reader 2; high magnification: $p<0.001$ for both readers) and was comparable to that of chromoendoscopy.

Conclusion: Both low-magnification and high-magnification NBI were capable of distinguishing neoplastic from non-neoplastic colorectal lesions; the diagnostic accuracy of NBI was better than that of conventional colonoscopy and equivalent to that of chromoendoscopy. The role of NBI in screening colonoscopy needs further evaluation.
\end{abstract}

M ost of the colorectal cancers (CRCs) arise from preexisting adenomas and such an adenoma-carcinoma sequence provides an opportunity for screening and prevention of CRC. ${ }^{1}$ Accumulating evidence has indicated that early detection and removal of colorectal adenomas may greatly reduce the mortality and incidence of $\mathrm{CRC}$, and reliable detection and resection of colorectal adenomas before they become malignant has a pivotal role in CRC screening. ${ }^{2}$ In the diagnosis of colorectal neoplasia, it is crucial to discriminate neoplastic lesions, including adenomas with dysplasia and invasive cancer, from non-neoplastic ones such as hyperplastic polyps to avoid overlooking neoplastic lesions and the risk of overtreatment. However, using conventional videoendoscopy, it is difficult to differentiate between neoplastic and nonneoplastic lesions and several new diagnostic modalities and endoscopic techniques have been developed to provide more precise diagnosis. Furthermore, previous studies have reported that the average size of depressed advanced lesions (high-grade dysplasia and cancer) is considerably smaller than that of polypoid lesions, showing that lesions should not be excluded based on small size. ${ }^{34}$

In chromoendoscopy, a biocompatible dye, such as indigo carmine, can strengthen the surface structure of epithelial lesions. Magnifying endoscopy allows more detailed observation of colour variation and fine structural change of the colonic mucosa. ${ }^{56}$ Various data have been reported on the diagnostic abilities of conventional colonoscopy, chromoendoscopy and magnifying endoscopy. ${ }^{6-11}$ Narrow-band imaging (NBI), developed by Gono et al, ${ }^{12}$ is a novel, non-invasive optical technique that uses reflected light to visualise the superficial structure and enhance vasculature within the mucosal layer. Gono et al reported that NBI clearly enhances the epithelial microvascular pattern. Thus, NBI can be used to differentiate between neoplastic and non-neoplastic gastrointestinal lesions. Yoshida et $a l^{13}$ and Muto et al ${ }^{14}$ reported that NBI allows detection of morphological changes in microvascular structure that are useful in the diagnosis of superficial oesophageal carcinomas. Machida et $a l^{15}$ demonstrated that NBI is equivalent to magnifying endoscopy for distinguishing colonic neoplasm from non-neoplastic lesions. Our study aimed to prospectively elucidate the diagnostic accuracy of NBI, under both low and high magnification, in distinguishing colorectal lesions and compare this accuracy to the diagnostic accuracies of conventional colonoscopy and of low-magnification and high-magnification chromoendoscopy.

\section{METHODS}

\section{Patients}

From December 2005 to March 2006, consecutive individuals who received total colonoscopy concurrently with conventional colonoscopy and NBI at National Taiwan University Hospital, Taipei, Taiwan and E-Da Hospital, Kaohsiung, Taiwan, were considered eligible for enrolment. This study was approved by the Institutional Review Board of both institutions.

Abbreviations: $C R C$, colorectal cancer; $N B I$, narrow-band imaging; NPV, negative predictive value; PPV, positive predictive value 


\section{Narrow-band imaging}

The NBI system has been described in detail in previous studies. The NBI system is based on modification of the spectral features with each optical filter narrowing a bandwidth of spectral transmittance. In this system, the central wavelengths of the dedicated trichromatic optical filters were 500, 445 and $415 \mathrm{~nm}$ and each had a bandwidth of $30 \mathrm{~nm}$.

A standard videoendoscopy system (EVIS LUCERA system, Olympus Optical, Tokyo, Japan) with two light sources was used for examination. One light source was for the standard optical filter (broadband) and the other was for the NBI system. The control knob on the grip of the endoscope allows singletouch exchange of the standard filter for the NBI filter. All endoscopic images were stored as electronic files in a digital image filing system for later analysis (Electronic Business Machines Technologies, EBM, Taipei, Taiwan).

\section{Colonoscopy}

Polyethylene glycol lavage solution (2 l; Klean-Prep, Harefield, Middlesex, UK) was used for colon preparation followed by a glycerin enema according to guidelines for preparation of all lower gastrointestinal examinations. Total colonoscopy was performed prospectively with an Olympus CF-Q240ZI colonoscope (Olympus Optical) by four experienced endoscopists (CHM, CCY, LYC and LWC); each of them had previously carried out a minimum of 2000 colonoscopies. Each detected lesion was irrigated without any residual faecal material on it before endoscopic observation. Endoscopic pictures for each lesion were taken in the order of conventional colonoscopy, low-magnification NBI, high-magnification NBI, low-magnification chromoendoscopy and high-magnification chromoendoscopy. After completion of NBI, chromoendoscopy was performed using $0.2 \%$ indigo carmine. A standard optical filter was used for conventional colonoscopy and chromoendoscopy. For diagnosis using chromoendoscopy, Kudo's classification of mucosal pit pattern was used; lesions with type III (including $\mathrm{III}_{\mathrm{L}}$ and $\left.\mathrm{III}_{\mathrm{S}}\right), \mathrm{IV}$ and $\mathrm{V}\left(\mathrm{V}_{\mathrm{I}}\right.$ and $\left.\mathrm{V}_{\mathrm{N}}\right)$ pits patterns were considered neoplastic. ${ }^{16}{ }^{17}$ For diagnosis using NBI, a brown blob under low magnification and a homogenous, dense vascular meshwork under high magnification were considered characteristic for neoplastic lesions (fig 1).

Biopsy, polypectomy or endoscopic mucosal resection was performed as indicated. Location of colorectal lesions was defined according to anatomical distribution. The caecum, ascending colon, hepatic flexure, transverse colon, and splenic flexure were defined as the proximal colon, whereas the descending colon and sigmoid colon were defined as the distal colon. All endoscopic specimens were evaluated by a single experienced pathologist (SCT), who was completely blind to endoscopic diagnosis. Histopathological diagnosis was based on the World Health Organization (WHO) criteria.

\section{Image evaluation}

All images were sorted by a fifth endoscopist (CCC), who was completely blinded to the endoscopic and pathological diagnoses of the lesions. The chart numbers of the patients on the endoscopic images were all processed and masked. Two experienced colonoscopists from National Taiwan University Hospital and E-Da Hospital (CHM and CCY) participated in the image reading and both readers were blinded to the histological diagnosis of all lesions.

We first conducted a substudy for assessment of interobserver variability using 10 images randomly selected from each diagnostic modality (conventional colonoscopy, low-magnification NBI, high-magnification NBI, low-magnification chromoendoscopy and high-magnification chromoendoscopy) and arranged randomly. Interobserver agreement on discriminating neoplastic from non-neoplastic lesions was assessed.

A total of 900 images of 180 lesions (each lesion was photographed during conventional colonoscopy, low-magnification NBI, high-magnification NBI, low-magnification chromoendoscopy and high-magnification chromoendoscopy) were selected for best image quality. Using random numbers, these 900 pictures were arranged in random order for readers 1 and 2 . Diagnostic accuracies of the different endoscopic modalities were determined by reference to the histopathological diagnosis.

\section{Statistical analysis}

For each of the different diagnostic modalities, sensitivities, specificities, positive predictive values (PPV), negative predictive values (NPV), diagnostic accuracies, and likelihood ratios were calculated. PPV was defined as (true positive/(true positive + false positive)), with true positive being the lesions that histopathology characterised as neoplastic and that the image reader evaluated as neoplastic. False-positive lesions are lesions that are non-neoplastic according to histopathology but that the reader diagnosed as neoplastic. NPV was defined as (true negative/(true negative + false negative)), with true negative being the lesions that histopathology characterised as non-neoplastic and that the reader evaluated as non-neoplastic. False-negative lesions are lesions that are neoplastic according to histopathology but that the reader diagnosed as nonneoplastic. Diagnostic accuracy was defined as ((true positive + true negative)/total number of cases). Likelihood ratio was defined as (sensitivity/( 1 -specificity)). Statistical differences of diagnostic accuracies were analysed by the $\chi^{2}$ test of independence or Fisher's exact test. $\mathrm{p}<0.05$ was considered significant. Statistical power was calculated for comparing conventional white light endoscopy with either low-magnification or highmagnification NBI and chromoendoscopy. Interobserver agreement for diagnosing colorectal lesions in the substudy was determined by calculation of $\kappa$ values and $95 \%$ CIs. A $\kappa$ value below 0.4 was regarded as representing poor agreement, a $\kappa$ value of $0.41-0.60$ fair agreement, a $\kappa$ value of $0.61-0.80$ good agreement, and a $\kappa$ value greater than 0.80 excellent agreement. Statistical analyses were conducted using SAS, V.9.0.

\section{RESULTS}

From December 2005 to March 2006, we screened 715 consecutive patients by total colonoscopy at the National Taiwan University Hospital and E-Da Hospital; we detected a total of 180 colorectal lesions in 133 patients. Table 1 shows the demographics of the patients and clinicopathological characteristics of the lesions.

Males represented $56.4 \%(n=75)$ of the cohort. Most lesions were observed in the proximal $(\mathrm{n}=74)$ and distal colon $(\mathrm{n}=81)$. Most of the lesions were classified as 0-Is $(119 / 180$ or $66.1 \%$ ) or 0 -IIa (49/180 or $27.2 \%)$. Histopathologically, 39 of the lesions were non-neoplastic and the other 141 lesions were neoplastic. No adverse events were reported.

There was excellent interobserver agreement in the sub-study ( $\kappa=0.86,95 \%$ CI 0.70 to 1 ) for all endoscopic modalities.

Figure 2 lists the representative images of a 0 -IIa lesion as viewed by the different endoscopic modalities. Table 2 summarises true-positive, true-negative, false-positive, and falsenegative findings, as well as sensitivities, specificities, diagnostic accuracies, PPVs, NPVs, and likelihood ratios of the different endoscopic modalities. Low-magnification NBI was less sensitive but had higher specificity than conventional colonoscopy. Low-magnification chromoendoscopy was more sensitive than low-magnification NBI. Under high 


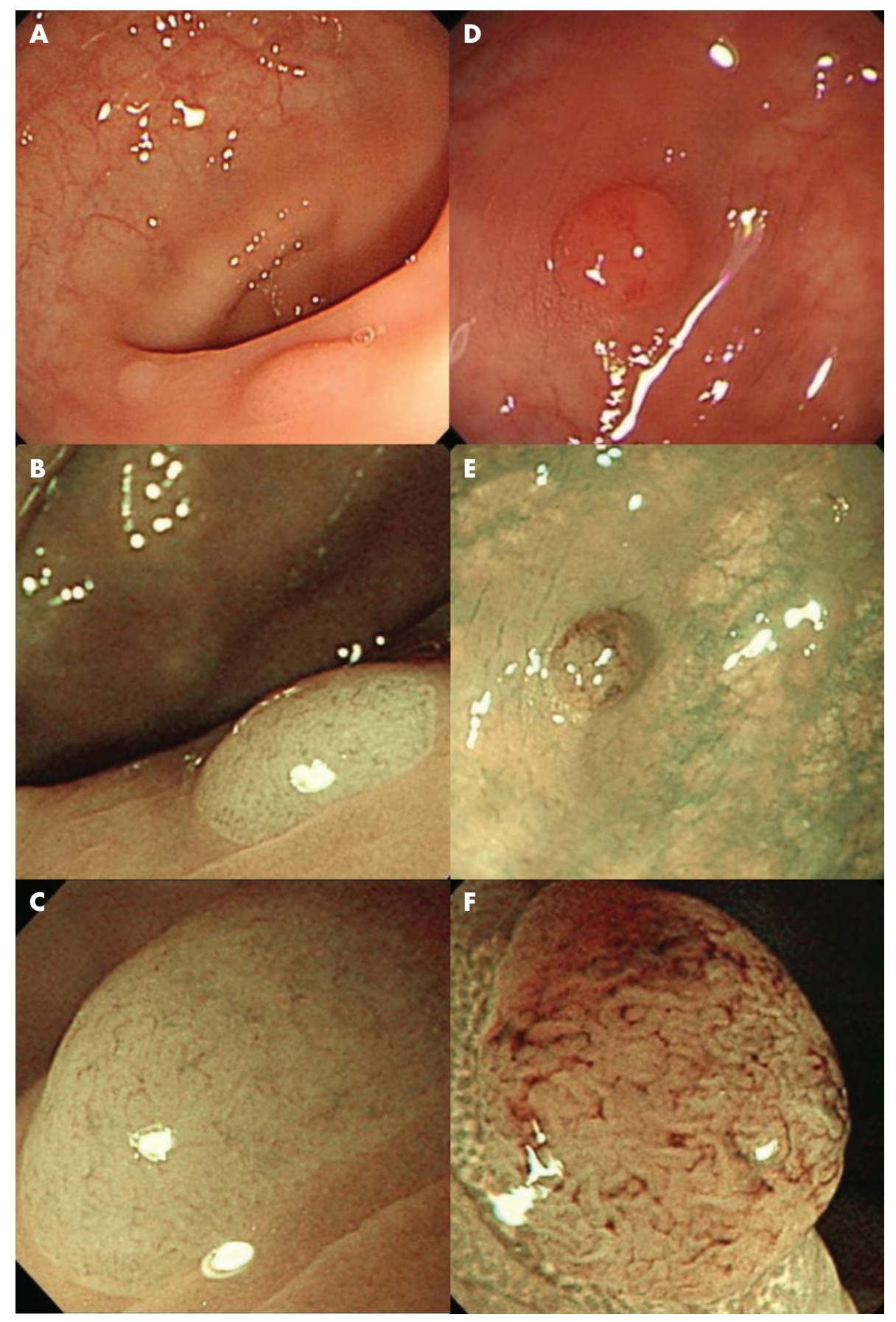

Figure 1 Use of narrow-band imaging (NBI) to distinguish between non-neoplastic and neoplastic colorectal lesions. (A)

Conventional view of a hyperplastic polyp. (B) Low-magnification NBI view of a hyperplastic polyp. (C) High-magnification $\mathrm{NBI}$ showed only minute thin vessels in a hyperplastic polyp. (D) Conventional view of an adenoma. (E) Low-magnification NB showed an adenoma as a characteristic brown blob. (F) High-magnification NBI showed a typical vascular network in an adenoma.

magnification, both sensitivity and specificity were considerably higher for NBI than for conventional colonoscopy, whereas sensitivity and specificity did not differ much between NBI and chromoendoscopy.

Diagnostic accuracies significantly improved when lowmagnification NBI $(82.4 \%$ for reader $\mathrm{l} \quad(\mathrm{p}=0.043$, power $=87 \%)$ and $80.6 \%$ for reader $2 \quad(p=0.004$, power $=82 \%)$ ) and low-magnification chromoendoscopy $(85.0 \%$ for reader $\mathrm{l}(\mathrm{p}=0.001$, power $=99 \%)$ and $78.9 \%$ for reader $2(p=0.013$, power $=99 \%))$, respectively, were compared with conventional colonoscopy. Diagnostic accuracies also considerably improved when high-magnification NBI $(87.2 \%$ for reader $1 \quad(p<0.001)$ and $90.0 \%$ for reader 2 $(\mathrm{p}<0.001))$ and high-magnification chromoendoscopy $(91.1 \%$ for reader $1 \quad(p<0.001)$ and $92.2 \%$ for reader $2 \quad(p<0.001))$, respectively, were compared with conventional colonoscopy. There was no significant difference of diagnostic accuracy when comparing low-magnification NBI with low-magnification chromoendoscopy $(p=0.0783$ for reader $\mathrm{l}$ and $\mathrm{p}=0.694 \mathrm{l}$ for reader 2), or high-magnification NBI with high-magnification chromoendoscopy ( $\mathrm{p}=0.4588$ for both reader 1 and reader 2 ).

\section{DISCUSSION}

According to previous pathological studies, the adenoma shares many architectural features with the carcinoma in terms of vascular architecture including vessel diameter and spatial distribution which is considerably different from that in the non-neoplastic portion of colonic mucosa. ${ }^{19}$ By using this 
Table 1 Demographics of study participants and clinicopathological characteristics of study lesions

\begin{tabular}{|c|c|}
\hline \multicolumn{2}{|l|}{ Patients $(n=133$ ) } \\
\hline Sex (male:female) & $75: 58$ \\
\hline Age, years (range) & $54.6(29-87)$ \\
\hline \multicolumn{2}{|l|}{ Lesions $(n=180)$} \\
\hline \multicolumn{2}{|l|}{ Location } \\
\hline Proximal colon & 74 \\
\hline Distal colon & 81 \\
\hline Rectum & 25 \\
\hline \multicolumn{2}{|l|}{ Morphology* } \\
\hline $0-1 p$ & 11 \\
\hline $0-\mathrm{Is}_{\mathrm{s}}$ & 119 \\
\hline 0-lia & 49 \\
\hline Advanced & 1 \\
\hline Mean size, $\mathrm{mm}$ (range) & $5.33(2-20)$ \\
\hline \multicolumn{2}{|l|}{ Histopathology } \\
\hline \multicolumn{2}{|l|}{ Non-neoplastic $(n=39)$} \\
\hline Hyperplastic polyp & 35 \\
\hline Inflammatory polyp & 3 \\
\hline Juvenile polyp & 1 \\
\hline \multicolumn{2}{|l|}{ Neoplastic $(n=141)$} \\
\hline Tubular adenoma & 134 \\
\hline Tubulovillous adenoma & 4 \\
\hline Villous adenoma & 1 \\
\hline Serrated adenoma & 1 \\
\hline Invasive cancer & 1 \\
\hline
\end{tabular}

characteristic angiogenesis during the process of tumorigenesis and the different penetration ability of different wavelengths of light, the concept of the NBI system successfully achieved enhancement and real-time observation of microvascular architecture in the gastrointestinal mucosal layer. ${ }^{12}$ In the NBI system, the central wavelengths of the trichromatic optical filters are 500, 445 and $415 \mathrm{~nm}$; each has a 30-nm bandwidth, with their spectral features corresponding to a penetration depth of 240, 200 and $170 \mu \mathrm{m}$, respectively. The thickness of gastrointestinal mucosa is around $700-800 \mathrm{~nm}$ and the unique characteristics of the NBI system make the observation of enhanced capillary structure within the mucosal layer possible; it provides endoscopists with the ability to differentiate neoplastic from non-neoplastic colorectal lesions with only a one-touch operation in real-time fashion.

According to a previous pilot study by Machida et $a l,{ }^{15}$ the NBI system has as high as $93.4 \%$ diagnostic accuracy, which is equivalent to chromoendoscopy with magnification, and its diagnostic accuracy is much higher than that of conventional colonoscopy. In their study, however, no data about diagnostic accuracy of NBI without magnification or chromoendoscopy without magnification were reported. We reported the data obtained from observation under both NBI and chromoendoscopy with or without magnification. This again emphasises the importance of contrast imaging, either with dye or digital means in the accurate diagnosis of colorectal neoplasia regardless of magnification observation. In fact, although recently more popular, magnifying colonoscopy is not standard endoscopic equipment in most institutions, not only in our country but also in Japan and Western countries. An endoscopy system installed with the NBI system enables the clinician to improve diagnostic accuracy and probably raise the detection rate of colon neoplasia. Another strength of our study is that we stored all the endoscopic images during examination and selected one picture from each of the five conditions of endoscopic observation and allocated randomly for diagnosis. This process decreased the observational bias and strengthened the reliability of our result because the findings on NBI pictures and chromoendoscopic images might influence each other and make objective evaluation of individual diagnostic modality difficult.
The classification of mucosal pit pattern of colorectal neoplasia has been established since its introduction in 1994 by Kudo et al. ${ }^{16}$ According to the standard classification criteria, neoplastic and non-neoplastic lesions could be discriminated easily by different pit pattern (type I and II are non-neoplastic and type III, IV and V are neoplastic) with accuracy as high as $90 \% .{ }^{8}$ This classification system has been applied in clinical use during the last 10 years and is well accepted. ${ }^{81020}$ On the contrary, well-accepted diagnostic criteria for observation of colorectal lesions using the NBI system are lacking and remain to be established. Although Machida et $a l^{15}$ indicated the characteristic appearance of a neoplastic lesion as a brown blob under low-magnification view and a unique vascular network with good to excellent surface pits visibility under high magnification, few equivocal pictures were observed in our study including some hyperplastic polyps or juvenile polyps that showed as a brown blob under low magnification and a vascular network under high magnification. According to our data, NBI with magnification yielded a higher specificity whereas chromoendoscopy with magnification yielded a higher sensitivity. This implies that these two modalities might compensate for each other and thus further improve diagnostic accuracy. In clinical practice, it is possible to initially detect a colorectal lesion under conventional view or low magnification with NBI mode. After identifying the lesion, further observation with the NBI system under high-magnification view followed by chromoendoscopy may increase diagnostic accuracy. Another advantage of NBI observation is its convenience without the necessity of dye spraying even though its diagnostic accuracy is the same as chromoendoscopy, and thus the procedural time can be shortened and an overlooked lesion with underaccumulation of dark-blue dye at the dependent portion of colon can also be avoided.

Although the usefulness of magnifying observation for discriminating neoplastic from non-neoplastic colorectal lesions and prediction of submucosal invasion has been reported in many studies, the longer vending part of magnifying colonoscopy and the resultant difficulty in insertion prevents it from being an all-pervading procedure, especially in the screening setting. Although the Lucera system is equipped with electric enhancement function without magnifying endoscopy, the upper limit of this function is $\times 1.8$ magnification which is still far from the maximal magnitude of magnification that magnifying endoscopy facilitates. Whether this magnification is adequate for discrimination of neoplastic from non-neoplastic lesions is still not clear and requires further study. A newly released magnifying colonoscopy device is thinner than the previous one (diameter of vending part is $14.8 \mathrm{~mm}$ for CFQ240Z and $13.6 \mathrm{~mm}$ for CFH260AZ; Olympus Optical) and is equipped with variable stiffness function that facilitates easier insertion, although objective data are still lacking.

In our study, two observers gained different degrees of increased diagnostic accuracy using different magnification of individual modalities. Although both observers had highly improved diagnostic accuracy using both low-magnification and high-magnification NBI or chromoendoscopy in comparison with conventional colonoscopy, it is not clear whether lowmagnification NBI could improve the detection rate in the mass population screening setting; this requires further study and investigation. The diagnostic sensitivity, specificity and accuracy comparing the conventional view with chromoendoscopy or with NBI varies in different studies. ${ }^{8}{ }^{15} 21$ This may be influenced by the characteristics of target lesions studied in individual studies. Specificity and negative predictive value might be higher if more hyperplastic polyps and more smallsized lesions were included in the study because most of the hyperplastic polyps are tiny and $<5 \mathrm{~mm}$ in size. ${ }^{8}$ Further study 


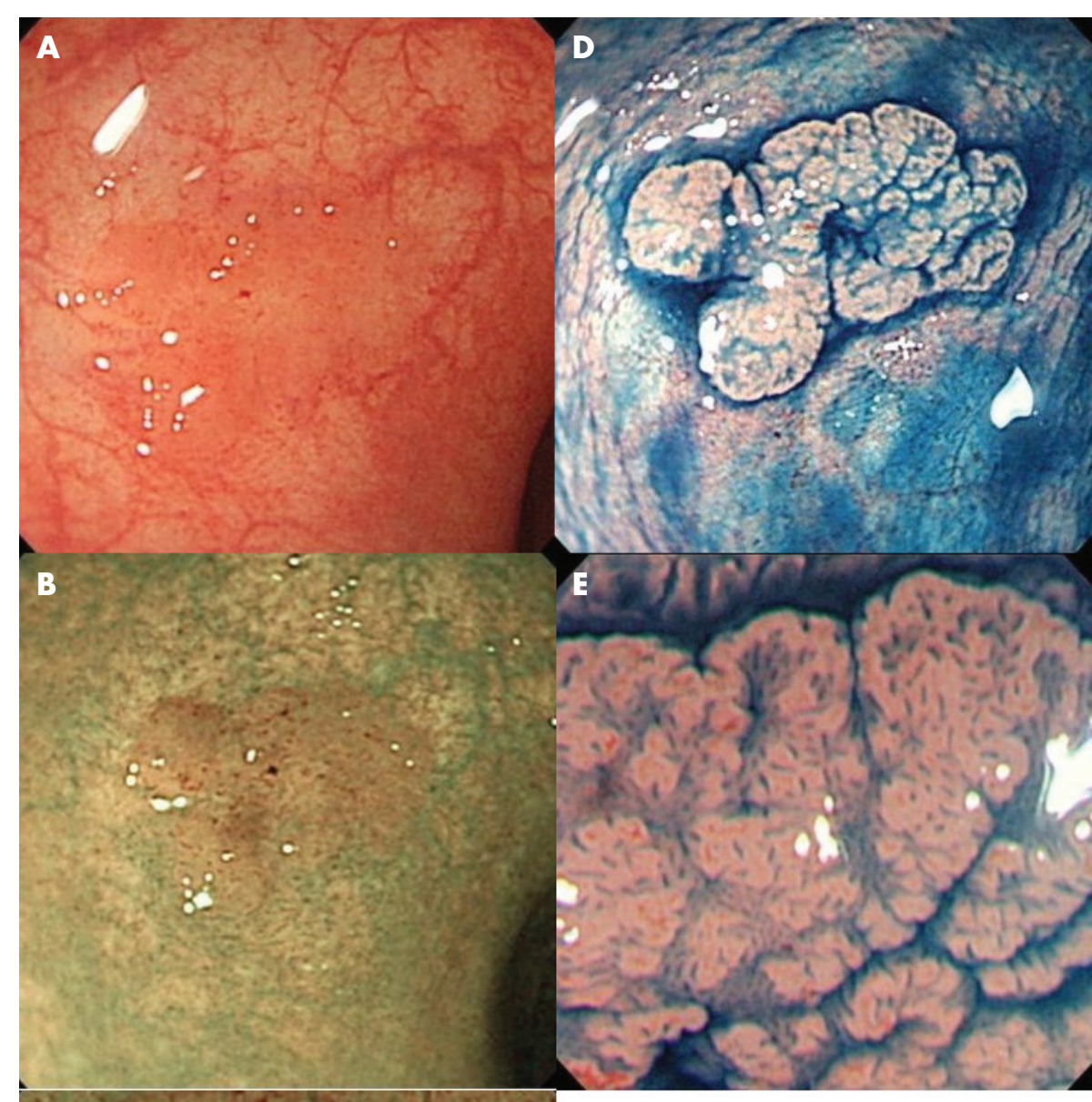

Figure 2 A 0-lla lesion viewed by the different endoscopic modalities used in this study. (A) Conventional colonoscopic view. (B) Low-magnification NBI showed a brown blob with scattered focal dense brown "stain". (C) High-magnification NBI showed a vascular network. (D) Low-magnification chromoendoscopy using $0.2 \%$ indigo carmine dye spraying clearly demarcated margins and surface structure. (E) Highmagnification chromoendoscopy clearly showed the mixed distribution of type IIIL and Ills pits.

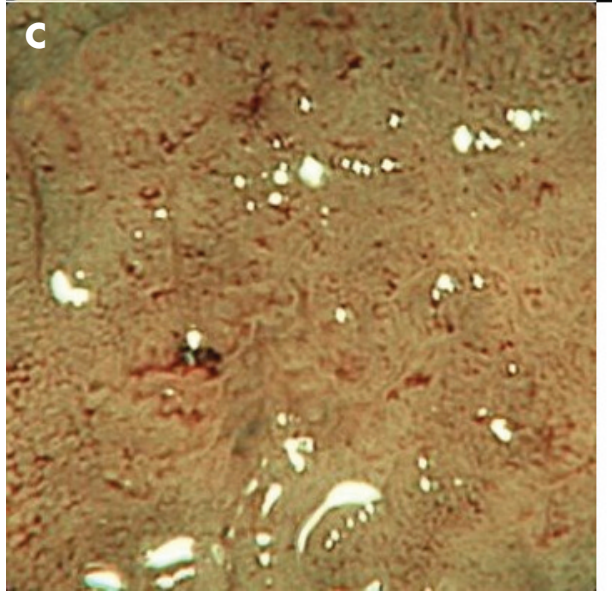

limiting target lesions to smaller ones using these diagnostic modalities is necessary to clarify their usefulness in small lesions in order to eliminate overtreatment and unnecessary biopsy. In addition, it is possible that endoscopists who are familiar with chromoendoscopic observation may also improve their own diagnostic accuracy with conventional colonoscopic observation but this requires further investigation.

There are several limitations to our study. First, we conducted this study using endoscopic photographs instead of video. In reality, an endoscopist usually views a detected lesion in a realtime fashion using multiple angles and light modalities at variable distances. Our design was aimed at reducing reading bias influenced by images taken using different diagnostic modalities to compare individual modalities independently and this was achieved at a cost of underestimation of diagnostic sensitivity, specificity, and accuracy. Another point worth mentioning is that our study was conducted using a nonhigh-definition system. The newly developed high-definition magnification system may show mucosal surface structures, including vascular architecture or pit pattern, more clearly during NBI or chromoendoscopic observation. Our study, which used a non-high-definition system, might underestimate the effect of these modalities, though there is still the possibility of improvement of diagnostic accuracy using conventional observation concurrently. To elucidate these points, further welldesigned studies are necessary. 
In summary, we demonstrated the high diagnostic accuracy of the NBI system in comparison with conventional colonoscopy and chromoendoscopy. Although the available data are limited, the NBI system facilitates both conventional and magnifying colonoscopy in the diagnosis of colorectal lesions. Our results warrant further study of the NBI system to determine its diagnostic accuracy in screening or surveillance colonoscopy in well-designed randomised trials, to assess its ability to diagnose the depth of invasion of neoplastic lesions, as well as to establish diagnostic criteria or a classification system of vascular network pattern for increased diagnostic accuracy.

\section{ACKNOWLEDGEMENTS}

We thank Dr WC Liao, Ms HY Yang and staff of the Health Management Center (National Taiwan University Hospital), as well as Drs $\mathrm{CH} \mathrm{Tu}$, CM Tai, CT Lee, and TH Chiang (E-Da Hospital, I-Shou University) for their invaluable help with the endoscopic procedures.

\section{Authors' affiliations}

Han-Mo Chiv, Chien-Chuan Chen, Yi-Chia Lee, Ming-Shiang Wu, JawTown Lin, Department of Internal Medicine, National Taiwan University Hospital, Taipei, Taiwan

Hsiu-Po Wang, Department of Emergency Medicine, National Taiwan University Hospital, Taipei, Taiwan

Chia-Tung Shun, Department of Pathology, National Taiwan University Hospital, Taipei, Taiwan

Chi-Yang Chang, Department of Internal Medicine, E-Da Hospital, I-Shou University, Kaohsiung, Taiwan

Competing interests: None.

\section{REFERENCES}

1 Vogelstein B, Fearon ER, Hamilton SR, et al. Genetic alterations during colorectal-tumor development. N Engl J Med 1988;319:525-32.

2 Winawer SJ, Zauber AG, Ho MN, et al. Prevention of colorectal cancer by colonoscopic polypectomy. The National Polyp Study Workgroup. N Engl J Med 1993:329:1977-81.

3 Saitoh Y, Waxman I, West AB, et al. Prevalence and distinctive biologic features of flat colorectal adenomas in a North American population. Gastroenterology 2001; 120:1657-65.

4 Tsuda S, Veress B, Toth E, et al. Flat and depressed colorectal tumours in a southern Swedish population: a prospective chromoendoscopic and histopathological study. Gut 2002;51:550-5.

5 Fujii T, Hasegawa RT, Saitoh Y, et al. Chromoscopy during colonoscopy. Endoscopy 2001;33:1036-41.

6 Hurlstone DP, Fujii T. Practical uses of chromoendoscopy and magnification at colonoscopy. Gastrointest Endosc Clin N Am 2005;15:687-702.

7 Konishi K, Kaneko K, Kurahashi T, et al. A comparison of magnifying and nonmagnifying colonoscopy for diagnosis of colorectal polyps: a prospective study. Gastrointest Endosc 2003;57:48-53.

8 Su MY, Ho YP, Chen PC, et al. Magnifying endoscopy with indigo carmine contrast for differential diagnosis of neoplastic and nonneoplastic colonic polyps. Dig Dis Sci 2004;49:1123-7.

9 Tanaka S, Haruma $K$, Ito $M$, et al. Detailed colonoscopy for detecting early superficial carcinoma: recent developments. J Gastroenterol 2000;35(Suppl 12): 121-5.

10 Nagata S, Tanaka S, Haruma K, et al. Pit pattern diagnosis of early colorectal carcinoma by magnifying colonoscopy: clinical and histological implications. Int J Oncol 2000;16:927-34.

11 Kiesslich R, von Bergh M, Hahn M, et al. Chromoendoscopy with indigocarmine improves the detection of adenomatous and nonadenomatous lesions in the colon. Endoscopy 2001;33:1001-6.

12 Gono K, Obi T, Yamaguchi M, et al. Appearance of enhanced tissue features in narrow-band endoscopic imaging. J Biomed Opt 2004;9:568-77.

13 Yoshida T, Inove H, Usui S, et al. Narrow-band imaging system with magnifying endoscopy for superficial esophageal lesions. Gastrointest Endosc 2004;59:288-95.

14 Muto M, Nakane M, Katada C, et al. Squamous cell carcinoma in situ at oropharyngeal and hypopharyngeal mucosal sites. Cancer 2004;101:1375-81.

15 Machida H, Sano Y, Hamamoto Y, et al. Narrow-band imaging in the diagnosis of colorectal mucosal lesions: a pilot study. Endoscopy 2004;36:1094-8.

16 Kudo S, Hirota S, Nakajima T, et al. Colorectal tumours and pit pattern. J Clin Pathol 1994;47:880-5. 
17 Kudo S, Rubio CA, Teixeira CR, et al. Pit pattern in colorectal neoplasia: endoscopic magnifying view. Endoscopy 2001;33:367-73.

18 Endoscopic Classification Review Group. Update on the Paris classification of superficial neoplastic lesions in the digestive tract. Endoscopy 2005;37:570-8.

19 Konerding MA, Fait E, Gaumann A. 3D microvascular architecture of precancerous lesions and invasive carcinomas of the colon. Br J Cancer $2001 ; 84: 1354-62$.
20 Kiesslich $\mathbf{R}$, Jung $M$, DiSario JA, et al. Perspectives of chromo and magnifying endoscopy: how, how much, when, and whom should we stain? J Clin Gastroenterol 2004;38:7-13.

21 Fu KI, Sano Y, Kato S, et al. Chromoendoscopy using indigo carmine dye spraying with magnifying observation is the most reliable method for differential diagnosis between non-neoplastic and neoplastic colorectal lesions: a prospective study. Endoscopy 2004;36:1089-93.

\section{EDITOR'S QUIZ: GI SNAPSHOTS}

\section{Malignant masquerade of the biliary confluence}

\section{Clinical presentation}

A 70-year-old man presented with a 6-week history of jaundice and pruritus that developed progressively. No previous medical history was noted. Physical examination showed a weight loss of $3 \mathrm{~kg} / 6$ weeks and jaundice. Biological tests showed cholestasis with $\gamma$-glutamyl transpeptidase levels at $604 \mathrm{IU} / \mathrm{l}$ (normal $<43 \mathrm{IU} / \mathrm{l}$ ), alkaline phosphatase levels at $443 \mathrm{IU} / \mathrm{l}$ (normal $<126 \mathrm{IU} / \mathrm{l})$, total bilirubin levels at $123 \mu \mathrm{m} / \mathrm{l}$ (normal $<22 \mu \mathrm{m} / \mathrm{l}$ ) and carbohydrate antigen 19-9 levels raised at $498 \mathrm{IU} / \mathrm{ml}$ (normal $<37 \mathrm{IU} / \mathrm{ml}$ ).

Abdominal ultrasonography showed dilated intrahepatic bile ducts and an acalculous non-distended gallbladder. Helical CT scan showed an obstruction at the hilar level with major upstream dilatation of the intrahepatic biliary tree without evidence of mass. Endoscopic retrograde cholangiopraphy was done (fig l).

\section{Question}

What is the diagnosis and management?

See page 389 for answer

R Noun, S Zeidan Department of Digestive Surgery, Hotel Dieu de France Hospital, Beirut, Lebanon C Ghorra Department of Pathology, Hotel Dieu de France Hospital, Beirut, Lebanon C Tohme-Noun Department of Radiology, Levant Hospital, Beirut, Lebanon
Robin Spiller, Editor

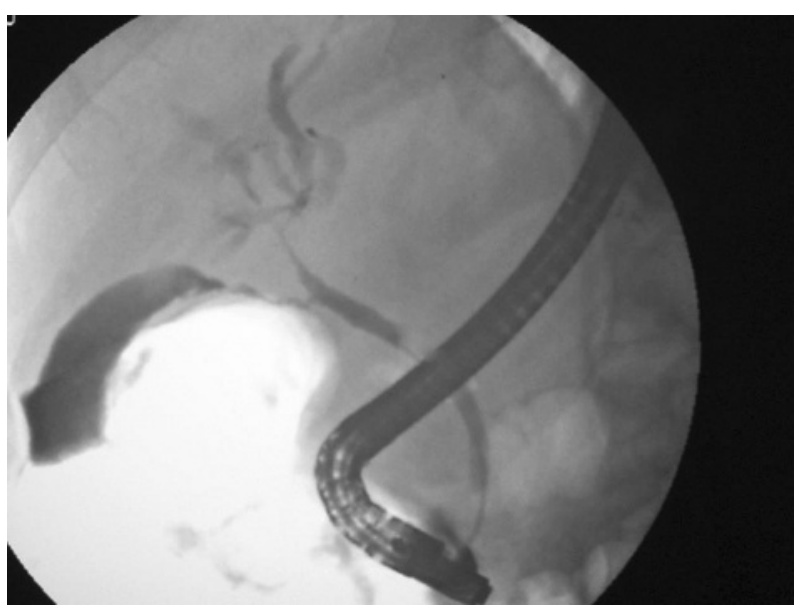

Figure 1 A tight stenosis of the hepatic hilum with dilatation of the intrahepatic ducts.

Correspondence to: Dr S Zeidan, Department of Digestive Surgery, Hotel Dieu de France Hospital, Alfred Naccache street, Achrafieh, PO Box 166830, Beirut, Lebanon; smart_zeidan@yahoo.com doi: 10.1136/gut.2006.095398 